\title{
Joint Outage Probability: An Exact Formulation for the Outage Problem in Wireless Systems
}

\author{
Flávio du Pin Calmon and Michel Daoud Yacoub
}

\begin{abstract}
Resumo-Este artigo apresenta uma nova formulação para a probabilidade de outage em sistemas de comunicação limitados por interferência, denominada Probabilidade de Outage Conjunta (JOP). Dado um conjunto de restrições para a relação sinal interferência de um grupo de sinais mutuamente interferentes, a JOP corresponde à probabilidade que ao menos uma restrição não seja satisfeita. Uma solução geral e exata para a probabilidade de outage conjunta é obtida, junto com uma condição necessária e suficiente para uma JOP não-nula. Além disso, uma formulação fechada para a JOP em um ambiente Rayleigh é encontrada. Os resultados apresentados podem ser utilizados diretamente em diversos cenários práticos.
\end{abstract}

Palavras-Chave-Probabilidade de Outage, Sistemas Limitados por Interferência, Relação Sinal-Interferência, Rayleigh.

Abstract-This paper presents a useful, novel formulation for the outage probability in interference-limited communication systems, here named Joint Outage Probability (JOP). Given a set of SIR restrictions for mutually interfering signals, the JOP corresponds to the probability that at least one of the restrictions is not satisfied. A general exact solution for the joint outage probability is derived, along with a necessary and sufficient condition for a non-null JOP. Furthermore, a closedform expression for the joint outage probability in a nonidentically distributed Rayleigh scenario with independent signals is obtained. The results presented here can be directly applied in a wide range of practical scenarios.

Keywords-Outage Probability, Signal-to-Interference Ratio, Interference-limited Systems, Rayleigh.

\section{INTRODUCTION}

Outage probability is a key figure of merit in wireless communications. In interference-limited systems, outage probability is commonly defined as the probability that the signalto-interference ratio (SIR) of a received signal is below a given threshold [1], [2]. Such a metric is directly related to system capacity in spread spectrum schemes, such as CDMA systems [3]. Furthermore, dense wireless networks, such as sensor and ad hoc networks, are intrinsically interference-limited, having the outage probability as a fundamental parameter for system analysis, design, and implementation [4]-[6].

The difficulty of the analysis of an outage condition may vary drastically. In some situations, it may be as simple as obtaining the probability of the occurrence of a single, straightforward event. In some others, it may involve the calculation of the probability of joint, intricate events. For instance, in call admission problems it may be desirable to

The authors are with the Wireless Technology Laboratory (WissTek), Department of Communications, School of Electrical and Computer Engineering, State University of Campinas (UNICAMP), P.O. Box 6101, 13083-852, Campinas, SP, Brazil (E-mail: [fcalmon,michel]@wisstek.org). This work was partly supported by FAPESP (07/02117-1). have a call admitted if, with such an admission, the interference experienced by all of the calls (i.e. those in progress as well as the one entering the system) remains under a certain tolerable threshold. In case the channels are independent and identically distributed (i.i.d.) and the threshold conditions are the same for all of the conversations (communications), one may lay hold of a symmetry property to simplify this problem and find an approximate solution as follows. Considering each channel to be identically affected by all of the others, then it approximately suffices to test the outage condition of any arbitrary individual channel with the admission of the call. But as said, this is an approximation even to the i.i.d. case. In addition, in practical situations and in multi-rate, multi-service networks, the channels are affected differently and the interference threshold may vary for each communication. Therefore, an exact solution for this general case is of paramount interest.

In light of the previous discussion, we present a useful, novel formulation for interference-limited communication systems, here named Joint Outage Probability (JOP). Given a set of SIR restrictions for $N$ mutually interfering signals with a certain joint distribution, the JOP corresponds to the probability that at least one of the restrictions is not satisfied or, dually, that all $N$ restrictions are attended. Hence, the JOP gives a direct measure of the outage probability for a group of signals detected by a receiver, not being limited to the outage of an individual signal. Although the results presented here have a theoretical nature, they can be directly applied in a wide range of practical scenarios, such as multiuser detection [7], power control [8], sensor positioning, wireless (multihop or not) network dimensioning [9] and, as hinted earlier, admission control problems [10]. To the best of the authors' knowledge, such a result is unprecedented in the literature.

The remainder of this paper is organized as follows. Section II presents a formal definition of the JOP problem. Section III derives the general, exact formulation to solve the JOP. In addition, a necessary and sufficient condition for a nontrivial JOP, dependent only on the SIR restrictions, and not on the signals' distribution, is found. Section IV presents a closed-form expression for the JOP in an independent non-identically Rayleigh distributed scenario with arbitrary interference thresholds. Finally, Section V presents some concluding remarks and a summary of the results.

\section{Outline of the Problem}

Let $W_{i}, i=1, \ldots, N$ be the instantaneous powers of interfering fading signals. In an interference-limited system, in order for the system to operate adequately, it is required that the signal-to-interference ratio at the receiver for any 
given signal $W_{i}$ be greater than a tolerable threshold $\beta_{i}$, $i=1, \ldots, N$, as specified for the particular communication. Therefore, the $N$ received signals must satisfy the set of inequalities (1), which describes a hypervolume in the $N$ dimensional space $\mathcal{S}^{N}$.

$$
\mathcal{S}^{N}=\left\{\begin{array}{c}
\frac{W_{1}}{\sum_{i=2}^{N} W_{i}} \geq \beta_{1} \\
\frac{W_{2}}{\sum_{\substack{i=1 \\
i \neq 2}}^{N} W_{i}} \geq \beta_{2} \\
\vdots \\
\frac{W_{N}}{\sum_{i=1}^{N-1} W_{i}} \geq \beta_{N}
\end{array}\right.
$$

We define as Joint Outage Probability $\left(P_{I}\right)$ the probability that at least one of the inequalities in (1) is not satisfied. The value of $P_{I}$ can be obtained as

$$
P_{\bar{I}}=\int_{\mathcal{S}^{N}} f_{\mathbf{W}}\left(w_{1}, \ldots, w_{N}\right) d w_{1} \ldots d w_{N},
$$

where $f_{\mathbf{W}}\left(w_{1}, \ldots, w_{N}\right)$ is the joint probability density function of $W_{1}, \ldots, W_{N}$ and $P_{\bar{I}}=1-P_{I}$ is the probability that all the inequalities in (1) are satisfied.

\section{THE EXACT SOLUTION FOR THE JOP}

In this section, the region $\mathcal{S}^{N}$ will be reformulated so as to make the integration in (2) tractable. This is done through four steps: (i) $\mathcal{S}^{N}$ will be divided into $N-1$ regions, denoted by $\mathcal{S}_{j}^{N}, j=1, \ldots, N-1$, each of which with well-defined integration limits; (ii) the union $\bigcup_{j=1}^{N-1} \mathcal{S}_{j}^{N}$ is shown to be equivalent to $\mathcal{S}^{N}$; (iii) $\mathcal{S}_{i}^{N}$ and $\mathcal{S}_{j}^{N}(j \neq i)$ are proved to form distinct integration regions; (iv) as a consequence of the previous steps, the right side of (2) will then be rewritten as the sum of $N-1$ integrals. The section is then finalized by determining the condition for a non-trivial $\mathcal{S}^{N}$ hypervolume.

\section{A. Dividing $\mathcal{S}^{N}$ into $N-1$ Regions}

The restrictions in (1) can be rearranged in terms of the $W_{N}$ as:

$$
\left\{\begin{aligned}
W_{N} & \leq \frac{W_{1}}{\beta_{1}}-\sum_{i=2}^{N-1} W_{i} \\
W_{N} & \leq \frac{W_{2}}{\beta_{2}}-\sum_{\substack{i=1 \\
i \neq 2}}^{N-1} W_{i} \\
& \vdots \\
W_{N} & \leq \frac{W_{N-1}}{\beta_{N-1}}-\sum_{i=1}^{N-2} W_{i} \\
W_{N} & \geq \beta_{N} \sum_{i=1}^{N-1} W_{i}
\end{aligned}\right.
$$

Noting that the first $N-1$ inequalities and the last inequality in (3) determine, respectively, upper and lower bounds for the value of $W_{N}$, (3) can be rewritten as

$$
\beta_{N} \sum_{i=1}^{N-1} W_{i} \leq W_{N} \leq \min _{1 \leq k \leq N-1}\left(\frac{W_{k}}{\beta_{k}}-\sum_{\substack{i=1 \\ i \neq k}}^{N-1} W_{i}\right) .
$$

(Of course, from (3) and (4), null values of $\beta_{k}$ will not impose an upper limit to $W_{N}$. With this observation in mind, and without loss of generality, we shall consider $\beta_{k} \neq 0$ for all $k$.)

Analyzing the upper limit for $W_{N}$ in (4), we find that $k=j$, $1 \leq j \leq N-1$, when

$$
W_{j} \leq \frac{\beta_{j}\left(1+\beta_{i}\right)}{\beta_{i}\left(1+\beta_{j}\right)} W_{i} \quad \forall i<N
$$

Consider a region such that (5) holds. Then (4) is rewritten as

$$
\beta_{N} \sum_{i=1}^{N-1} W_{i} \leq W_{N} \leq \frac{W_{j}}{\beta_{j}}-\sum_{\substack{i=1 \\ i \neq j}}^{N-1} W_{i}
$$

A necessary condition for (6) to be non-trivial is

$$
\frac{\left(1-\beta_{j} \beta_{N}\right)}{\beta_{j}\left(1+\beta_{N}\right)} W_{j} \geq \sum_{\substack{i=1 \\ i \neq j}}^{N-1} W_{i}
$$

For convenience, we define the constants

$$
\begin{aligned}
\mathbb{K}_{j, k} & =\frac{\beta_{j}\left(1+\beta_{k}\right)}{\beta_{k}\left(1+\beta_{j}\right)} \\
\mathbb{C}_{j, N} & =\frac{\left(1-\beta_{j} \beta_{N}\right)}{\beta_{j}\left(1+\beta_{N}\right)} .
\end{aligned}
$$

Combining (5) to (7), the following restriction is found for $W_{N-1}$ :

$$
\mathbb{K}_{N-1, j} W_{j} \leq W_{N-1} \leq \mathbb{C}_{j, N} W_{j}-\sum_{\substack{i=1 \\ i \neq j}}^{N-2} W_{i}
$$

In the same way, for $W_{N-2}$,

$$
\mathbb{K}_{N-2, j} W_{j} \leq W_{N-2} \leq \mathbb{C}_{j, N} W_{j}-\sum_{\substack{i=1 \\ i \neq j}}^{N-3} W_{i}-W_{N-1}
$$

Using the lower bound for $W_{N-1}$ in (10), (11) can be rewritten as

$$
\mathbb{K}_{N-2, j} W_{j} \leq W_{N-2} \leq\left(\mathbb{C}_{j, N}-\mathbb{K}_{N-1, j}\right) W_{j}-\sum_{\substack{i=1 \\ i \neq j}}^{N-3} W_{i}
$$

Proceeding in a similar manner for $W_{N-3}, W_{N-4}, \ldots, W_{1}$, we find the following set of inequalities, which describe a 
region defined as $\mathcal{S}_{j}^{N}$ :

$$
\left\{\begin{array}{c}
\beta_{N} \sum_{i=1}^{N-1} W_{i} \leq W_{N} \leq \frac{W_{j}}{\beta_{j}}-\sum_{\substack{i=1 \\
i \neq j}}^{N-1} W_{i} \\
\mathbb{K}_{N-1, j} W_{j} \leq W_{N-1} \leq \mathbb{C}_{j, N} W_{j}-\sum_{\substack{i=1 \\
i \neq j}}^{N-2} W_{i} \\
\vdots \\
\quad \vdots \quad \mathbb{K}_{k, j} W_{j} \leq W_{k} \leq\left(\mathbb{C}_{j, N}-\sum_{\substack{i=k+1 \\
i \neq j}}^{N-1} \mathbb{K}_{i, j}\right) W_{j}-\sum_{\substack{i=1 \\
i \neq j}}^{k-1} W_{i} \\
\vdots \\
\mathbb{K}_{1, j} W_{j} \leq W_{1} \leq\left(\mathbb{C}_{j, N}-\sum_{\substack{i=2 \\
i \neq j}}^{N-1} \mathbb{K}_{i, j}\right) W_{j} \\
0 \leq W_{j}<\infty
\end{array}\right.
$$

$$
\text { B. Proof that } \bigcup_{j=1}^{N-1} \mathcal{S}_{j}^{N}=\mathcal{S}^{N}
$$

Next, we prove that the region formed by the union $\bigcup_{j=1}^{N-1} \mathcal{S}_{j}^{N}$ is equal to that of $\mathcal{S}^{N}$. From the derivation of $\mathcal{S}_{j}^{N}$, it is clear that an $N$-tuple $\left(W_{1}, W_{2}, \ldots, W_{N}\right)$ belonging to $\mathcal{S}^{N}$, with the $j$ th element, $1 \leq j \leq N-1$, satisfying (5), will also be contained in $\mathcal{S}_{j}^{N}$. We note that, in case $\left(W_{1}, W_{2}, \ldots, W_{N}\right) \in \mathcal{S}^{N}$, there will always be at least one element that satisfies (5), since the upper limit of (4) necessarily has a minimum. If $m$ elements satisfy (5), it is straightforward to show that the $N$-tuple will belong to $m$ regions of the form $\mathcal{S}_{j}^{N}$. It follows that any given $N$-tuple belonging to $\mathcal{S}^{N}$ will also be contained in $\bigcup_{j=1}^{N-1} \mathcal{S}_{j}^{N}$.

The converse can be proved by contradiction. Assume that an $N$-tuple $\left(W_{1}, W_{2}, \ldots, W_{N}\right)$ belonging to $\bigcup_{j=1}^{N-1} \mathcal{S}_{j}^{N}$ exists that does not satisfy at least one of the inequalities in (3). Then, we may consider that, for some $k \neq N$,

$$
W_{N}>\frac{W_{k}}{\beta_{k}}-\sum_{\substack{i=1 \\ i \neq k}}^{N-1} W_{i} .
$$

In case $W_{k}$ satisfies (5), (14) confutes the first inequality in (13), contradicting the initial assumption. Otherwise, suppose, without loss of generality, that condition (5) holds for $j \neq k$. Thus,

$$
\begin{aligned}
\frac{W_{k}}{\beta_{k}} & <W_{N}+\sum_{\substack{i=1 \\
i \neq k}}^{N-1} W_{i} \\
& \leq \frac{W_{j}}{\beta_{j}}-\sum_{\substack{i=1 \\
i \neq j}}^{N-1} W_{i}+\sum_{\substack{i=1 \\
i \neq k}}^{N-1} W_{i} \\
\Rightarrow W_{k} & <\mathbb{K}_{k, j} W_{j},
\end{aligned}
$$

contradicting the fact that $W_{j}$ satisfies (5).
When $k=N$, we have

$$
W_{N}<\beta_{N} \sum_{i=1}^{N-1} W_{i}
$$

contradicting the first inequality in (13). Therefore, an element belonging to the region $\bigcup_{j=1}^{N-1} \mathcal{S}_{j}^{N}$ will also belong to $\mathcal{S}^{N}$.

\section{Proof that $\mathcal{S}_{j}^{N}$ and $\mathcal{S}_{k}^{N}$ Form Distinct Integration Regions}

We will prove that the $N$-dimensional hypervolume of $\mathcal{S}_{j}^{N} \cap \mathcal{S}_{k}^{N}(j \neq k)$ is zero, implying that $\mathcal{S}_{j}^{N}$ and $\mathcal{S}_{k}^{N}$ are distinct integration regions. In case an element $\left(W_{1}, W_{2}, \ldots, W_{N}\right)$ belongs to both $\mathcal{S}_{j}^{N}$ and $\mathcal{S}_{k}^{N}$, we find from (5) that

$$
W_{j}=\mathbb{K}_{j, k} W_{k} \text {. }
$$

Since two elements are dependent, the fact that the $N$ dimensional hypervolume is null follows directly. Intuitively, this means that the regions $\mathcal{S}_{j}^{N}$ and $\mathcal{S}_{k}^{N}$ have only "border" points in common, with disjoint interiors. Therefore, the integration region defined by $\mathcal{S}_{j}^{N}$ does not overlap with the one defined by $\mathcal{S}_{k}^{N}$.

\section{Main Result}

Based on the previous developments, (2) can be rewritten as

$$
P_{\bar{I}}=\sum_{j=1}^{N-1} \int_{\mathcal{S}_{j}^{N}} f_{\mathbf{W}}\left(w_{1}, \ldots, w_{N}\right) d w_{1} \ldots d w_{N},
$$

or, equivalently,

$$
\begin{aligned}
& P_{\bar{I}}=\sum_{j=1}^{N-1} \int_{0}^{\infty} \int_{\mathbb{K}_{1, j} w_{j}}^{\left(\mathbb{C}_{j, N}-\sum_{\substack{i=2 \\
i \neq j}}^{N-1} \mathbb{K}_{i, j}\right) w_{j}} \cdots \\
& \times \int_{\mathbb{K}_{j-1, j} w_{j}}^{\left(\mathbb{C}_{j, N}-\sum_{i=j+1}^{N-1} \mathbb{K}_{i, j}\right) w_{j}-\sum_{i=1}^{j-2} w_{i}} \int_{\mathbb{K}_{j+1, j} w_{j}}^{\left(\mathbb{C}_{j, N}-\sum_{i=j+2}^{N-1} \mathbb{K}_{i, j}\right) w_{j}-\sum_{i=1}^{j-1} w_{i}} \ldots \\
& \times \int_{\mathbb{K}_{N-1, j} w_{j}}^{\mathbb{C}_{j, N} w_{j}-\sum_{\substack{i=1 \\
i \neq j}}^{N-2} w_{i}} \int_{\beta_{N} \sum_{i=1}^{N-1} w_{i}}^{\substack{w_{j} \\
\beta_{j}} \sum_{\substack{i=1 \\
i \neq j}}^{N-1} w_{i}} f_{\mathbf{W}}\left(w_{1}, w_{2}, \ldots, w_{N}\right) \\
& \times d w_{N} d w_{N-1} \ldots d w_{j+1} d w_{j-1} \ldots d w_{1} d w_{j} .
\end{aligned}
$$

We note that the index $j$ of the summation terms in (16) and (17) will only span the values for which $\beta_{j} \neq 0$.

\section{E. The Condition for a Non-Trivial $\mathcal{S}^{N}$}

From (17), a necessary and sufficient condition for the integration region defined in (1) to be a non-trivial one is

$$
\sum_{i=1}^{N} \frac{\beta_{i}}{1+\beta_{i}}<1 \text {. }
$$

This is proved as follows. In order for (13) to define a nontrivial integration region, the upper limit must be necessarily larger than the lower limit for every inequality. Therefore, from 
the restriction for $W_{1}$ in (13) (or equivalently for $W_{2}$ in case $\mathcal{S}_{1}^{N}$ is considered), we have that

$$
\mathbb{C}_{j, N}>\sum_{\substack{i=1 \\ i \neq j}}^{N-1} \mathbb{K}_{i, j}
$$

We will proceed by induction to show that (19) is also a sufficient condition for the integration region $\mathcal{S}_{j}^{N}$ to be nontrivial. Assume that (19) is satisfied, which is the base case of the inductive argument. Suppose, also, that the upper limit of the inequality corresponding to $W_{k}$ in (13) is necessarily larger than the lower one. We consider, without loss of generality, that $k \neq j-1$. Therefore,

$$
\begin{aligned}
\mathbb{K}_{k, j} W_{j}<\left(\mathbb{C}_{j, N}-\sum_{\substack{i=k+1 \\
i \neq j}}^{N-1} \mathbb{K}_{i, j}\right) W_{j}-\sum_{\substack{i=1 \\
i \neq j}}^{k-1} W_{i} \\
\Rightarrow \sum_{\substack{i=1 \\
i \neq j}}^{k-1} W_{i}<\left(\mathbb{C}_{j, N}-\sum_{\substack{i=k \\
i \neq j}}^{N-1} \mathbb{K}_{i, j}\right) W_{j} .
\end{aligned}
$$

Analyzing the upper limit for $k+1$ :

$$
\begin{aligned}
\left(\mathbb{C}_{j, N}-\sum_{\substack{i=k+2 \\
i \neq j}}^{N-1} \mathbb{K}_{i, j}\right) W_{j}-\sum_{\substack{i=1 \\
i \neq j}}^{k} W_{i} & >\left(\mathbb{K}_{k, j}+\mathbb{K}_{k+1, j}\right) W_{j}-W_{k} \\
& \geq \mathbb{K}_{k+1, j} W_{j}
\end{aligned}
$$

The first inequality follows directly from (20), whereas the second one is justified by (5), proving the induction step. Thus, if the condition for $W_{1}$ is satisfied in (13), the restriction for $W_{2}, W_{3}, \ldots, W_{N-1}$ will be non-trivial.

Finally, to prove that $W_{N}$ will also have a non-trivial restriction when (19) is satisfied, we note that its limits in (13) depend on all the other $N-1$ variables. Consequently, it must be shown that $W_{1}, W_{2}, \ldots, W_{N-1}$ can assume values such that

$$
\mathbb{C}_{j, N} W_{j}>\sum_{\substack{i=1 \\ i \neq j}}^{N-1} W_{i}
$$

where the inequality follows from (7). However, given (19), (22) will be satisfied when $W_{1}, W_{2}, \ldots, W_{N-1}$ assume their minimum values in (13), which concludes the proof. The restriction (18) follows directly by summing $1+\mathbb{K}_{N, j}$ to both sides of (19).

It is interesting to note that (18) is independent of $\mathcal{S}_{j}^{N}$, even though a specific region was considered in the proof. Furthermore, (18) depends solely on the SIR thresholds, and not on the signals' distribution. This fact explicits that, even though the inequalities in (1) may have a non-null probability of being satisfied individually, there will always be at least one signal outage unless (18) is attended.

\section{General, Closed-Form Expression FOR A RAYLEIGH ENVIRONMENT}

In this section, a closed-form expression for the joint outage probability in a Rayleigh environment is derived, assuming independent interfering signals. The probability density function of $W_{i}$ is given by

$$
f_{W_{i}}\left(w_{i}\right)=\frac{1}{\Omega_{i}} \exp \left(-\frac{w_{i}}{\Omega_{i}}\right),
$$

where $\Omega_{i}$ is the mean power, and the joint density distribution of $W_{1}, W_{2}, \ldots, W_{N}$ is the product

$$
f_{\mathbf{W}}\left(w_{1}, w_{2}, \ldots, w_{N}\right)=\prod_{i=1}^{N} f_{W_{i}}\left(w_{i}\right) .
$$

The integration of the joint distribution (24) presents a regularity, since it is the product of exponential functions. As a consequence, for a fixed $j$ in the summation term, the innermost integrals of (17) have a pattern, denoted by $\varphi_{j}(\cdot)$. For $j=1$ in $(17), \varphi_{1}(\cdot)$ is given by

$$
\begin{aligned}
& \varphi_{1}(r, s, \boldsymbol{\Omega}, \boldsymbol{\beta}, c)=\sum_{i=N-c}^{N} \frac{\left(1+\beta_{N}\right) \Omega_{N} \Omega_{i}^{c}}{\left(\beta_{N} \Omega_{i}+\Omega_{N}\right) \prod_{\substack{j=N-c \\
j \neq i}}^{N}\left(\Omega_{i}-\Omega_{j}\right)} \\
& \times\left(\exp \left(-\frac{(r+s) \beta_{N}}{\Omega_{N}}-r \sum_{j=N-c}^{N-1} \frac{\left(\Omega_{N}+\beta_{N} \Omega_{j}\right)}{\Omega_{j} \Omega_{N}} \mathbb{K}_{j, 1}\right)\right. \\
& \left.\left.-\exp \left(\frac{\beta_{i} s-r}{\beta_{i} \Omega_{i}}+r \sum_{\substack{j=N-c \\
j \neq i}}^{N} \frac{\left(\Omega_{j}-\Omega_{i}\right)}{\Omega_{j} \Omega_{i}} \mathbb{K}_{j, 1}\right)\right), \quad(2)\right)
\end{aligned}
$$

where, $r$ and $s$ are auxiliary variables, respectively, denoting $w_{j}$ and the sum of the remaining $w_{i}$ which will be posteriorly integrated; $\boldsymbol{\Omega}=\left(\Omega_{1}, \Omega_{2}, \ldots, \Omega_{N}\right) ; \boldsymbol{\beta}=\left(\beta_{1}, \beta_{2}, \ldots, \beta_{N}\right)$; and $\varphi_{1}(\cdot)$ satisfies

$\varphi_{1}(r, s, \boldsymbol{\Omega}, \boldsymbol{\beta}, 1)=\int_{\mathbb{K}_{N-1,1} r}^{\mathbb{C}_{1, N} r-s} \frac{e^{-\frac{t}{\Omega_{N-1}}}}{\Omega_{N-1}} \int_{\beta_{N}(r+s+t)}^{\frac{r}{\beta_{1}}-s-t} \frac{e^{-\frac{u}{\Omega_{N}}}}{\Omega_{N}} d u d t$

and

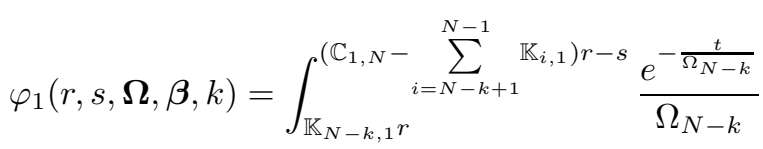

$$
\begin{aligned}
& \times \varphi(r, s+t, \boldsymbol{\Omega}, \boldsymbol{\beta}, k-1) d t .
\end{aligned}
$$

It is straightforward to show that (25) satisfies (26) and (27).

The value of $\varphi_{i}(\cdot)$ for $i \neq 1$, as well as the corresponding properties (26) and (27), can be obtained directly from (25) with the appropriate change of indexes. When two signals $W_{m}$ and $W_{n}$ have the same mean power, the corresponding expression for $\varphi_{1}(\cdot)$ can be found by taking the limit $\Omega_{m} \rightarrow$ $\Omega_{n}$ in (25).

Using (17) and the property (27) of $\varphi_{i}(\cdot)$, the value of $P_{\bar{I}}$ for the Rayleigh scenario under investigation is

$$
P_{\bar{I}}=\sum_{m=1}^{N-1} P_{\bar{I}, m}
$$

where

$$
P_{\bar{I}, m}=\int_{0}^{\infty} \frac{e^{-\frac{r}{\Omega_{m}}}}{\Omega_{m}} \varphi_{m}(r, 0, \boldsymbol{\Omega}, \boldsymbol{\beta}, N-2) d r .
$$


Evaluating the integral in (29), and after tedious and lengthy simplifications, $P_{\bar{I}, m}$ is found to be

$$
\begin{gathered}
P_{\bar{I}, m}=\frac{\left(\frac{\left(1+\beta_{N}\right) \beta_{m}}{1+\beta_{m}}\right)\left(1-\sum_{j=1}^{N} \frac{\beta_{j}}{1+\beta_{j}}\right)^{N-1}}{\left(\prod_{l=1}^{N} \Omega_{l}\right)\left(\sum_{j=1}^{N-1} \frac{\beta_{j}}{1+\beta_{j}}\left(\frac{\beta_{N} \Omega_{j}+\Omega_{N}}{\Omega_{j} \Omega_{N}}\right)\right)} \\
\times \prod_{\substack{i=1 \\
i \neq m}}^{N}\left(\frac{1}{\Omega_{i}}+\sum_{j=1}^{N} \frac{\beta_{j}\left(\Omega_{i}-\Omega_{j}\right)}{\left(1+\beta_{j}\right) \Omega_{i} \Omega_{j}}\right)^{-1} .
\end{gathered}
$$

Substituting (30) in (28) and performing further simplifications, we arrive at the final exact, closed-form expression for $P_{\bar{I}}$ :

$$
P_{\bar{I}}=\frac{\left(1-\sum_{j=1}^{N} \frac{\beta_{j}}{1+\beta_{j}}\right)^{N-1}}{\prod_{i=1}^{N}\left(1-\sum_{j=1}^{N} \frac{\beta_{j}}{1+\beta_{j}}\left(1-\frac{\Omega_{i}}{\Omega_{j}}\right)\right)} .
$$

This expression is general, and may be applied even when one or more signals have the same average power or a null restriction value, i.e., $\beta_{j}=0$. It is noteworthy that condition (18) appears explicitly in the numerator of (31).

When $\beta_{j}=\beta$ and $\Omega_{j}=\Omega$ for all $j$, (31) simplifies to

$$
P_{\bar{I}}=\left(\frac{1-(N-1) \beta}{1+\beta}\right)^{N-1} \text {. }
$$

Equation (32) is illustrated in Fig. 1.

\section{CONCLUSions}

In this paper, we presented a new formulation for interference-limited wireless systems, namely joint outage probability. An integral form for the JOP was derived in terms of the individual SIR restrictions and the signals' joint probability distribution. Through further analysis, a necessary and sufficient condition for a non-null JOP was found, which depends solely on the SIR restrictions. An application of these results led to an exact, simple, closed-form expression of the joint outage probability in a Rayleigh environment, considering independent signals. The formulations derived here are general, and may be applied in various practical scenarios.

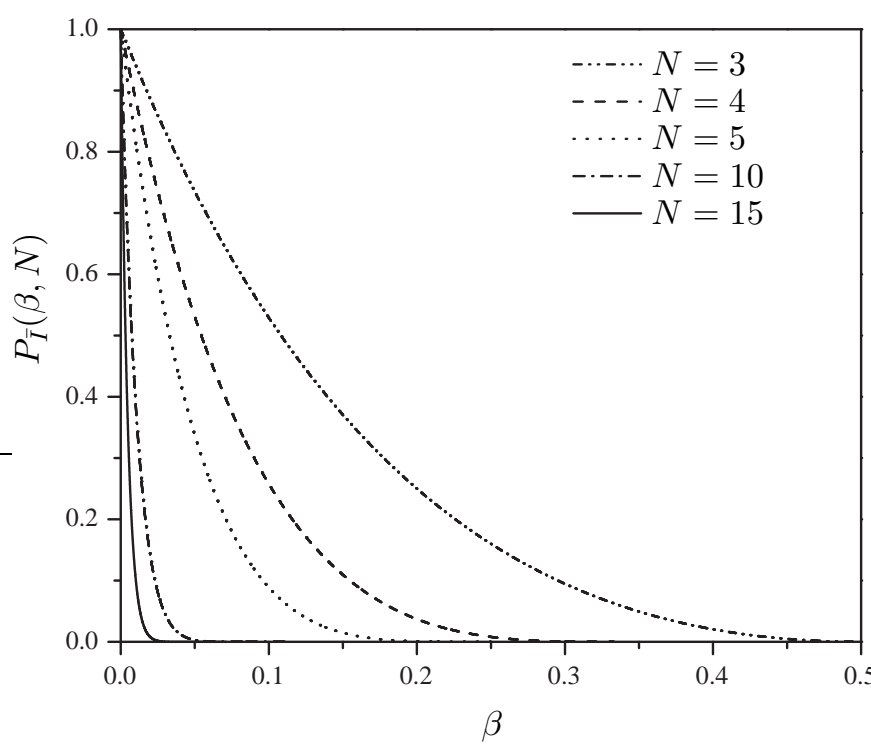

Fig. 1. $\quad P_{\bar{I}}$ for a Rayleigh i.i.d. environment with equal restrictions $\beta$.

\section{REFERENCES}

[1] M. D. Yacoub, Fundamentals of Mobile Radio Engineering, 1st ed. CRC-Press, Apr. 1993.

[2] A. J. Viterbi, CDMA: Principles of Spread Spectrum Communication. Prentice Hall PTR, Apr. 1995.

[3] K. Gilhousen, I. Jacobs, R. Padovani, A. Viterbi, L. Weaver, and C. Wheatley, "On the capacity of a cellular CDMA system," IEEE Trans. Veh. Technol., vol. 40, no. 2, pp. 303-312, May 1991.

[4] S. Weber, X. Yang, J. Andrews, and G. de Veciana, "Transmission capacity of wireless ad hoc networks with outage constraints," IEEE Trans. Inform. Theory, vol. 51, no. 12, pp. 4091-4102, Dec. 2005.

[5] J. Andrews, S. Weber, and M. Haenggi, "Ad hoc networks: To spread or not to spread?" IEEE Commun. Mag., vol. 45, no. 12, pp. 84-91, Dec. 2007.

[6] P. Gupta and P. Kumar, "The capacity of wireless networks," IEEE Trans. Inform. Theory, vol. 46, no. 2, pp. 388-404, Mar. 2000.

[7] J. Andrews, "Interference cancellation for cellular systems: a contemporary overview," IEEE Wireless Commun. Mag., vol. 12, no. 2, pp. 19-29, Apr. 2005.

[8] R. Yates, "A framework for uplink power control in cellular radio systems," IEEE J. Select. Areas Commun., vol. 13, no. 7, pp. 13411347 , Sept. 1995.

[9] A. A. M. de Medeiros and M. D. Yacoub, "An analytical approach for dimensioning wireless multihop networks," in IEEE Global Telecommun. Conf., Nov. 2007, pp. 4687-4691.

[10] S. Singh, V. Krishnamurthy, and H. Poor, "Integrated voice/data call admission control for wireless DS-CDMA systems," IEEE Trans. Signal Process., vol. 50, no. 6, pp. 1483-1495, June 2002. 